\title{
Comparison of Susceptibility of Various Candida Species Isolated From Neonatal Septicaemia to Voriconazole and Fluconazole
}

\author{
Kavitha $\mathrm{H}^{*}$ Anuradha K, Venkatesha D \\ Department of Microbiology, Mysore Medical College and Research Institute, Mysore-570001, Karnataka, \\ India.
}

\begin{abstract}
:
Background: Candida species are known to be the most common fungal pathogens isolated from blood cultures of neonates. Recent reports from our country indicates trend towards an increasing prevalence of non-albicans candidemia. Candida species possess a number of virulence factors which enable them to cause hematogenously disseminated infections in susceptible hosts with increased morbidity and mortality.

Objectives:To know the prevalence of candidemia in neonates and their antifungal susceptibility pattern. Materials and Methods: Blood samples from suspected cases of neonatal septicemia were subjected to culture, incubated for 7 days and subcultures performed. Culture yielding pure growth of Candida were included for the study and identified by standard methodology. Antifungal susceptibility was performed towards fluconazole and voriconazole by disc diffusion method on Muller-Hinton agar with 2\% glucose and methylene blue.

Result: Among 1583 blood cultures 80(5\%) candida isolates were obtained. The various species isolated were C.pseudotropicalis 34(42.5\%), C.tropicalis 22(27.5\%), C.albicans 12 (15\%) C.guillermondii 6(7.5\%), C.parapsilosis 4(5\%) and C.krusei 2(2.5\%). Fluconazole susceptibility was observed in 56(70\%) sensitive, 18 (22.5\%) Susceptible-Dose Dependent (SDD) and 6(7.5\%) resistant and all were sensitive to voriconazole. Conclusion: In this study non albicans candida was the common isolate \& they showed decreased resistance to Fluconazole. In neonatal septicemia speciation \& antifungal susceptibility may help in management.
\end{abstract}

Key words: candidemia,fluconazole, non-albicans candida,voriconazole

\section{Introduction}

Candida spp. is one of the most frequent pathogens isolated in bloodstream infections (BSI), and is associated with significant morbidity and mortality in hospitalized patients.[1][2][3] Candida spp. currently ranks as the fourth and the seventh most common bloodstream pathogen in North American and European studies.[4][5] Neonates and infants have been populations with some highest risk of candidemia.[6] Importance of Candida species in nursery and intensive care units (ICUs) is increasingly being recognized. Candida species account for 9-13\% of all blood isolates in neonatal intensive care units (NICUs).[7] Previous studies have suggested that possible risk factors such as common use of broad-spectrum antibiotics, low birth weight (LBW), prematurity, asphyxia neonatorum, hyperalimentation, presence of intravascular catheters, surgery, total parenteral nutrition and intensive care unit stay have made neonates prone to candidemia.[8][9][10][11] Historically C. albicans has been the most frequently isolated species worldwide.[8] However, over the last two decades, infections caused by the nonalbicans Candida have been diagnosed with increasing frequency, notably $\mathrm{C}$. tropicalis, C. glabrata, and C. parapsilosis.[9][10] There are published data from various centres regarding the incidence and relative frequency of Candida spp.[12]

The incidence and associated mortality due to candidemia can be influenced by several factors including characteristic of the population at risk, standard of the health care facilities available, distribution of Candida species, and prevalence of antifungal resistance. These factors may vary from one geographical region to other.[13] Fluconazole, offered an attractive alternative to amphotericin B in the treatment of candidaemia and various invasive candida infections. Major concerns about reduced fluconazole efficacy and increasing occurrence of Candida spp. potentially resistant to azole drugs led to development of new antifungal drugs. [14] The spectrum of activity of voriconazole gives it an inherent advantage over other antifungals. Voriconazole is active against all Candida species.[15] It is active against even those strains of Candida krusei and Candida glabrata that have been found to be fluconazole resistant, and strains of Candida albicans which demonstrate acquired resistance to fluconazole.[15][16] Amphotericin was compared with intravenous voriconazole for empiric fungal infection voriconazole was found to be superior with fewer side effects. Advantages include oral route of administration with wide spectrum of coverage without the renal and platelet lowering side effects. In addition, there is a significant cost advantage over liposomal amphotericin.[17] The increased isolation rates of nonalbicans Candida species and a gradual shift in the antifungal susceptibility profile have underlined the need 
to monitor laboratory data for possible emergence of resistance and to select most appropriate antifungal agent for therapy.

Hence, a prospective study was taken up to know the isolation rate, prevailing Candida spp and their anti-fungal susceptibility to fluconazole and voriconazole in neonates.

\section{Materials and Methods}

A total of 1583 blood samples from clinically suspected cases of neonatal septicemia were subjected to culture over a period of one year. The blood inoculated in to Brain Heart Infusion broth were incubated at $37^{\circ} \mathrm{C}$ and subcultured routinely onto Mac Conkey's medium \& Blood agar at 24 hours, $48 \mathrm{hrs}, 72 \mathrm{hrs}$ and on $7^{\text {th }}$ day. Whenever growth of white opaque colony on Blood agar was noticed, Gram's stain was performed to study the morphology, yeast like budding cells were further speciated by using germ-tube production, cornmeal agar morphology and sugar assimilation tests.[18] Anti-fungal susceptibility was done on Muller Hinton agar with $2 \%$ glucose and methylene blue $(0.5 \mu \mathrm{g} / \mathrm{ml})$ to fluconazole $(25 \mu \mathrm{g})$ and voriconazole $(1 \mu \mathrm{g})$ (HiMedia, Mumbai) using disc diffusion method. Zone diameters were interpreted as per the approved NCCLS (M44-A) guidelines.

\section{Results}

Culture with Candida as only isolates were included for the study. A total of 80 (5.05\%) Candida isolates were recovered from 1583 blood samples. Most of the isolates were non-albicans Candida (85\%). C. pseudotropicalis accounted for $42.5 \%$ of the isolates, followed by C. tropicalis $(27.5 \%)$, C. albicans (15\%), C. guillermondii (7.5\%), C. parapsilosis $(5 \%)$ and C. krusei $(2.5 \%)$.(Fig.1,2)

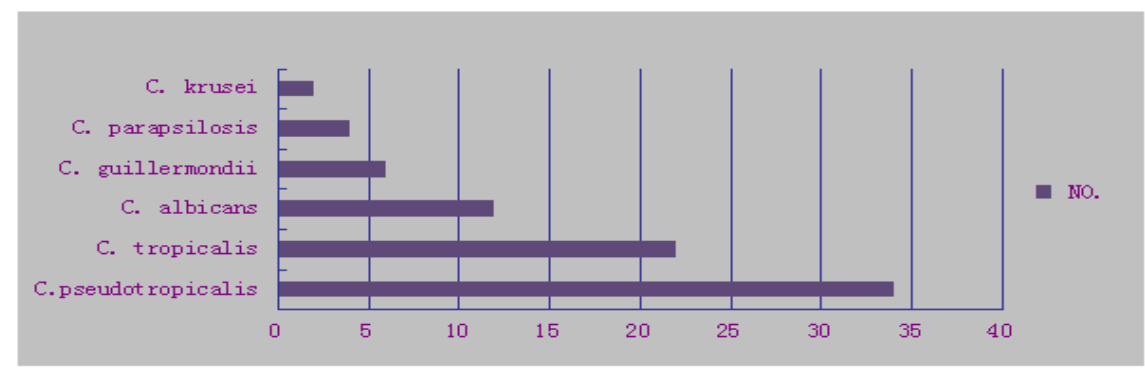

Fig.1: No. of different Candida species
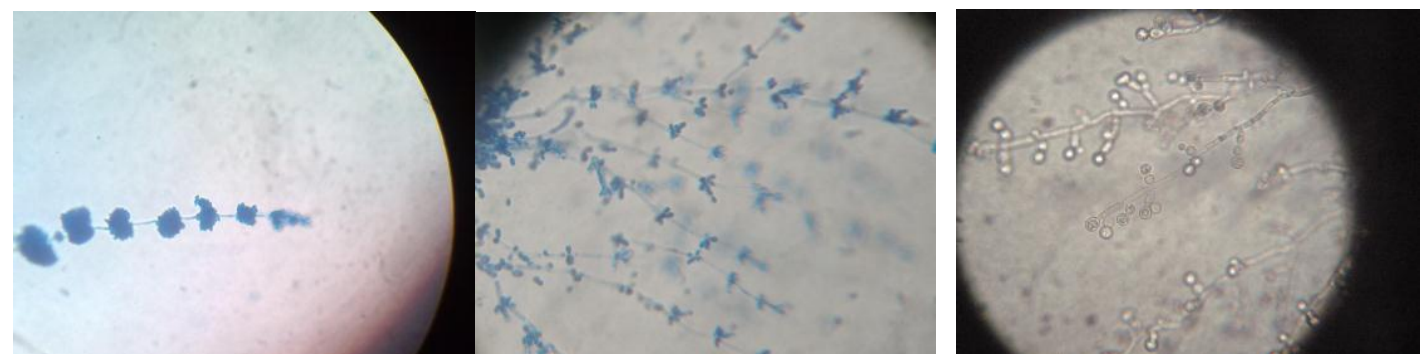

Fig.2: Microscopic picture of C. guillermondii, C. tropicalis and C. albicans

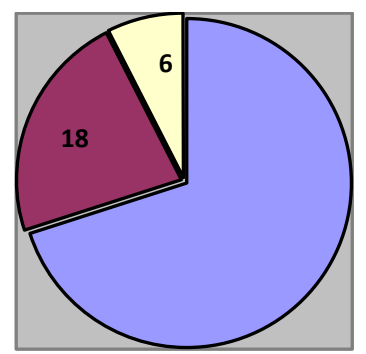

$\square$ sensitive $\square$ SDD $\square$ resistant

Fig.3: Fluconazole susceptibility of candida isolates 
The Candida isolates showed $100 \%$ sensitivity to voriconazole. Whereas susceptibility to fluconazole $56(70 \%)$ isolates were sensitive, $18(22.5 \%)$ were Susceptible-Dose Dependent (S-DD) and $6(7.5 \%)$ were resistant.(Fig.3)

Fluconazole susceptibility of various candida species, C.guillermondii and C. parapsilosis was found to be susceptible and C.krusei was found to be resistant, shown in Table1

Table 1: Fluconazole susceptibility of various Candida species

\begin{tabular}{|l|l|l|l|}
\hline Species & Sensitive & Susceptible-Dose Dependent & Resistant \\
\hline C. pseudotropicalis & $22(64.7 \%)$ & $12(35.3 \%)$ & - \\
\hline C.tropicalis & $14(63.63 \%)$ & $6(27.3 \%)$ & $2(9 \%)$ \\
\hline C.albicans & $10(83.33 \%)$ & - & $2(16.66 \%)$ \\
\hline C.guillermondii & $06(100 \%)$ & - & - \\
\hline C.parapsilosis & $04(100 \%)$ & - & - \\
\hline C.krusei & - & - & $2(100 \%)$ \\
\hline
\end{tabular}

\section{Discussion}

Neonatal candidemia has gained significant importance and has increased the concern in diagnosis of neonatal septicemia.There has been a surge of infections due to yeast infections other than C. albicans. [19] Infections with these yeast species also have a direct impact on the choice of empiric antifungal therapy and clinical outcome. The potential clinical importance of species-level identification has been recognized as Candida species differ in the expression of putative virulence factors and antifungal susceptibility.[20]

The prevalence rate of Candida species in neonatal septicemia was $5.05 \%$ which is supported by other studies[7][8] but lower than several reports showing frequency of isolation in 13.6-19.6\% cases.[9] In our study, non-albicans Candida blood stream infections (85\%) were more common than C. albicans blood stream infections. This finding is consistent with other studies where non-albicans Candida species predominate in Indian subcontinent, Asia and Europe.[12][21][22] Emergence of Candida spp. other than C. albicans is due to selection of less susceptible species by the pressure of antifungal agent such as fluconazole apart from associated risk factors and underlying disorders.[23]

C. pseudotropicalis (C. kefyr) $(42.5 \%)$ and C. tropicalis (27.5\%) are the common species responsible for neonatal candidemia in the present study. This is in contrast with other studies reporting C. tropicalis, C. parapsilosis and C. albicans as commonest.[3][8][12][24] However there are increased case reports showing that neonatal candida septicaemia is being caused by C. pseudotropicalis (C. kefyr) and other rare unspecified species.[25][26][27][28] The occurrence of C. kefyr fungemia reflects the growing diversity of Candida species responsible for disseminated infections in neutropenic patients.[29] We have isolated two C. krusei $(2.5 \%)$ in our study which is generally low in all settings and geographic regions.[22]

Antifungal susceptibility in our study revealed that the Candida isolates were $70 \%$ sensitive to fluconazole. Earlier Indian data show that fluconazole susceptibility to Candida were 75\%[12] and 95.53\%.[8] Twelve isolates of C. pseudotropicalis and six isolates of C. tropicalis were found to be in the Susceptible Dose Dependant (SDD) range $(15-18 \mathrm{~mm})$ to which voriconazole has demonstrated good in-vitro activity, correlating with Hazen et al.[30] All the isolates were $100 \%$ sensitive to voriconazole which is in line with other studies.[12][31] But a study has shown a very high resistance of 56\% to voriconazole.[24] Our study suggests that voriconazole might be effective in the treatment of refractory candidemia caused by fluconazole-resistant strains. With various types of antifungals available in the market, it has become necessary to perform anti-fungal susceptibility testing and reporting for effective therapeutic outcome. Evaluation of newer anti-fungal agents is needed.

Our study is limited by a single institution's experience, lack of follow-up and antifungal susceptibility testing performed only to azole group. Amphotericin B has not been included in our study even though it continues to be the standard therapy for systemic mycoses because its use is limited by severe side effects, mainly nephrotoxicity and high cost. Further multi-centric studies are required to know the epidemiology and drug resistance.

\section{Conclusion}

It is important to monitor distribution of Candida species and resistance trends in the face of increasing usage of potent, broad-spectrum antibacterial agents in hospitals. The changing epidemiology of candidaemia, highlights the need for close monitoring of Candida species distribution and perform antifungal susceptibility testing to develop local guidelines on empiric therapy for invasive candidiasis, based on the epidemiology of infection which will help us to recognize the emerging fungal pathogen and increasing drug resistance. 
We conclude that considering the in-vitro data, high oral bioavailability together with its pharmacokinetics, clinical efficacy and safety profile, voriconazole can be added to the treatment of fungal sepsis in newborns who still have persistent candidemia despite conventional antifungal management.

\section{References}

[1] Tortorano AM, Dho G, Prigitano A, Breda G, Grancini A, Emmi V, Cavanna C, Marino G, Morero S, Ossi C, Invasive fungal infections in the intensive care unit: a multicentre, prospective, observational study in Italy (2006-2008), Mycoses, 55(1), 2012, 73-79.

[2] Kourkoumpetis T, Manolakaki D, Velmahos G, Chang Y, Alam HB, De Moya MM, Sailhamer EA, Mylonakis E, Candida infection and colonization among non-trauma emergency surgery patients, Virulence, 1(5), 2010, 359.

[3] Shivaprakasha S, Radhakrishnan K, Karim P, Candida spp. other than Candida albicans: A major cause of fungaemia in a tertiary care centre, Indian J Med Microbiol, 25(4), 2007, 405-7.

[4] Wisplinghoff H, Bischoff T, Tallent SM, Seifert H, Wenzel RP, Edmond MB, Nosocomial bloodstream infections in US hospitals: analysis of 24,179 cases from a prospective nationwide surveillance study, Clin Infect Dis, 39(3), 2004, 309-317.

[5] Marchetti O, Bille J, Fluckiger U, Eggimann P, Ruef C, Garbino J, Calandra T, lauser MP, Täuber MG, Pittet D, Epidemiology of candidemia in Swiss tertiary care hospitals: secular trends, 1991-2000, Clin Infect Dis, 38(3), 2004, 311-320.

[6] Roilides E, Invasive candidiasis in neonates and children, Early Hum Dev, 87, 2011, S75-S76.

[7] Baradkar VP, Mathur M, Kumar S, Rathi M, Candida glabrata emerging pathogen in neonatal sepsis, Ann Trop Med Public Health, 1, 2008, 5-8.

[8] Nidhi Goel, Prabhat K Ranjan, Ritu Aggarwal, Uma Chaudhary, Nanda Sanjeev, Emergence of Nonalbicans Candida in Neonatal Septicemia and Antifungal Susceptibility: Experience from a Tertiary Care Center, J Lab Physicians, Jul-Dec, 1(2), 2009, 53-55.

[9] Agarwal J, Bansal S, Mailk GK, Jain A, Trends in neonatal septicemia: Emergence of non-albican Candida, Indian Pediatr,41, 2004, $712-5$.

[10] Nguyen MH, Peacock JE Jr, Morris AJ, Tanner DC, Minh LN, Snydman DR, et al ., The changing face of candidemia: Emergence of non- Candida albicans species and antifungal resistance, Am J Med , 100, 1996, 617-23.

[11] Fridkin SK, Jarvis WR, Epidemiology of nosocomial fungal infections, Clin Microbiol Rev, 9, 1996, $499-511$.

[12] Adhikary R, Joshi S, Species distribution and anti-fungal susceptibility of Candidaemia at a multi super-specialty center in Southern India, Indian J Med Microbiol , 29, 2011, 309-11.

[13] Hobson RP, The global epidemiology of invasive Candida infections: Is the tide turning? J Hosp Infect., 20, $2003,159-68$.

[14] Rene Pelletier, Line Loranger, Helene Marcotte, Emidio De Carolis, Voriconazole and fluconazole susceptibility of Candida isolates, J Med Microbiol, 51(6), 2002, 6 479-483

[15] Vikas Kohli, Vikas Taneja, Poonam Sachdev, Raja Joshi, Voriconazole in Newborns, Indian Pediatrics, 45, 2008, $236-238$.

[16] M H Nguyen, C Y Yu, Voriconazole against fluconazole-susceptible and resistant candida isolates: in-vitro efficacy compared with that of itraconazole and ketoconazole, J. Antimicrob. Chemother, 42 (2), 1998, 253-256.

[17] Walsh TJ, Pappas P, Winston DJ, Lazarus HM, Peterson F, Raffali J, et al., Voriconazole compared with liposomal amphotericin B for empirical anti-fungal therapy in patients with neutropenia and persistent fever, N Engl J Med, 346, 2002, $225-234$.

[18] Chander J, A text book of medical mycology, 3rd ed., Candidiasis (New Delhi: Mehta Publishers, 2009). 266-90.

[19] Agarwal S, Manchanda V, Verma N, Bhalla P, Yeast identification in routine clinical microbiology laboratory and its clinical relevance, Indian J Med Microbiol, 29, 2011, 172-7.

[20] Baillie GS, Douglas LJ, Iron-limited biofilms of Candida albicans and their susceptibility to amphotericin B, Antimicrob Agents Chemother ,42, 1998, 2146-9.

[21] Chakrabarti A, Mohan B, Shrivastava SK, Marak RS, Ghosh A, Ray P, Change in distribution and antifungal susceptibility of Candida species isolated from candidaemia cases in a tertiary care centre during 1996-2000, Indian J Med Res, 116, 2002, 5-12.

[22] Falagas ME, Roussos N, Vardakas KZ, Relative frequency of albicans and the various non-albicans Candida spp among candidemia isolates from inpatients in various parts of the world: A systematic review, Int J Infect Dis, 14, 2010, e954-66.

[23] Berrouane YF, Herwaldt LA, Pfaller MA, Trends in antifungal use and epidemiology of nosocomial yeast infections in a university hospital, J Clin Microbiol, 37, 1999, 531-7.

[24] Kothari A, Sagar V, Epidemiology of candida bloodstream infections in a tertiary care institute in India, Indian J Med Microbiol 27, 2009, 171-2.

[25] H E J Catfermole and R P A Rivers, Neonatal candida septicaemia: diagnosis on buffy smear, Archives of Disease in Childhood, 62, 1987.

[26] Stefan Weichert, Konrad Reinshagen, Katrin Zahn, Gernot Geginat, Annebärbel Dietz, Anna Kristina Kilian, Horst Schroten and Tobias Tenenbaum, Candidiasis caused by Candida kefyr in a neonate: Case report, BMC Infectious Diseases, 12, $2012,61$.

[27] Kevin C. Hazen, New and Emerging Yeast Pathogens, CLINICAL MICROBIOLOGY REVIEWS, Oct. 1995, $462-478$.

[28] D. J. Diekema, S. A. Messer, L. B. Boyken, R. J. Hollis, J. Kroeger, S. Tendolkar, and M. A. Pfaller, In Vitro Activity of Seven Systemically Active Antifungal Agents against a Large Global Collection of Rare Candida Species as Determined by CLSI Broth Microdilution Methods, J Clin Microbiol, 47(10), Oct 2009, 3170-3177.

[29] Christoph W. M. Reuter, Michael A. Morgan, Franz-Christoph Bange, Florian Gunzer, Matthias Eder, Bernd Hertenstein, Arnold Ganser, Candida kefyr as an Emerging Pathogen Causing Nosocomial Bloodstream Infections in Neutropenic Leukemia Patients, Clin Infect Dis, 41(9), 2005, 1365-1366.

[30] Kevin C. Hazen, Ellen Jo Baron, Arnaldo Lopes Colombo, Corrado Girmenia, Aurora Sanchez-Sousa, Amalia del Palacio, Catalina de Bedout, David L. Gibbs and The Global Antifungal Surveillance Group, Comparison of the Susceptibilities of Candida spp. to Fluconazole and Voriconazole in a 4-Year Global Evaluation Using Disk Diffusion, J. Clin. Microbiol, 41(12), 2003, 5623-5632.

[31] Chun-fang Ma, Fang-qiu Li1, Li-ning Shi, Yu-an Hu, Ying Wang, Mei Huang, Qian-qian Kong, Surveillance study of species distribution, antifungal susceptibility and mortality of nosocomial candidemia in a tertiary care hospital in China, BMC Infectious Diseases, 13, 2013, 337. 